\title{
Ventilatory Demand During Stepping and Running: Implications for Exercise-Induced Bronchoconstriction in Children
}

\author{
Jessyca P Selman, Fernanda C Lanza, Gustavo F Wandalsen, Dirceu Solé, Denis E O’Donnell, \\ J Alberto Neder, and Simone Dal Corso
}

\begin{abstract}
BACKGROUND: Single-step tests have been proposed as simple and inexpensive challenges to diagnose exercise-induced bronchoconstriction (EIB) in the pediatric population. Work performed and the resulting ventilation, however, might be substantially lower in stepping than running. This might decrease the diagnostic yield of step-based challenges. METHODS: In a cross-sectional study, 53 children with asthma with exercise-related symptoms (34 boys, age 6-18 y) underwent an incremental stepping test, a 6-min constant stepping test, and a treadmill running test on different days. RESULTS: Constant and incremental stepping tests presented with lower metabolic demands $\left(\dot{\mathrm{V}}_{\mathrm{O}_{2}} 1.42 \pm 0.48\right.$ and $1.34 \pm 0.55 \mathrm{~L} / \mathrm{min}$, respectively), ventilatory demands $\left(\dot{\mathrm{V}}_{\mathrm{O}_{2}} 45 \pm 14\right.$ and $43 \pm 16 \mathrm{~L} / \mathrm{min}$, respectively), and cardiovascular demands $(160 \pm 20$ and $161 \pm 19$ beats $/ \mathrm{min}$, respectively) than the treadmill running test $(1.65 \pm 0.60 \mathrm{~L} / \mathrm{min}, 54 \pm 17 \mathrm{~L} / \mathrm{min}$, and $172 \pm 7$ beats/min, respectively) $(P<.05)$. Between-test agreement in diagnosing EIB was poor (kappa 0.217-0.361). Although EIB prevalence was higher after the treadmill running test $(60 \%)$ compared to constant $(53 \%)$ and incremental $(47 \%)$ stepping tests, 7 subjects developed EIB only in stepping. Clinical and resting functional characteristics did not differ in discordant subjects (ie, EIB negative in a given test but positive in another) versus concordant subjects (ie, EIB negative or positive in both tests). EIB was not related to individual test ability in eliciting high to very-high ventilation ( $\geq 40 \%$ or $\geq 60 \%$ maximum voluntary ventilation, respectively). Moreover, a negative stepping test but a positive treadmill test (and vice versa) was not associated with greater ventilatory demands. CONCLUSIONS: Lower prevalence of EIB in stepping compared to treadmill running is not related to less ventilation demand in the former modality. Although stepping might be useful as a screening EIB test due its portability and low cost, a negative test should be confirmed with a running-based test in symptomatic children. Key words: exercise; ventilation; asthma; dyspnea; exercise-induced asthma; children. [Respir Care 2019;64(4):445-452. (c) 2019 Daedalus Enterprises]
\end{abstract}

\section{Introduction}

Exercise is a common and potent trigger of asthmatic symptoms in children. ${ }^{1}$ Exercise-induced bronchoconstric-

\footnotetext{
Ms Selman and Drs Lanza and Dal Corso are affiliated with the Postgraduate Program in Rehabilitation Sciences, Universidade Nove de Julho, São Paulo, Brazil. Drs Wandalsen and Solé are affiliated with the Division of Allergy, Clinical Immunology and Rheumatology, Department of Pediatrics, Federal University of São Paulo, São Paulo, Brazil. Drs O'Donnell and Neder are affiliated with the Laboratory of Clinical Exercise Physiology \& Respiratory Investigation Unit, Division of Respiratory and Critical Care Medicine, Queen's University and Kingston General Hospital, Kingston, Canada.
}

Ms Selman presented a version of this manuscript at the 17th Congresso tion (EIB) is a marker of inadequate disease control, and it is associated with poor quality of life and increased utilization of health care resources. ${ }^{2}$ EIB worldwide prevalence in children and adolescents with asthma is $46 \%^{3}$ Unfortunately, reported symptoms are imprecise to diagnose EIB, particularly in children. ${ }^{4,5}$ Thus, several exercise

Paulista de Pneumologia e Tisiologia, held November 15-18, 2017, in São Paulo, Brazil.

Ms Selman has disclosed a relationship with the São Paulo Research Foundation. The other authors have disclosed no conflicts of interest.

Supplementary material related to this paper is available at http:// www.rcjournal.com. 
challenge tests have been proposed to dehydrate and cool down the airways, thereby eliciting EIB in susceptible individuals. ${ }^{1,2,6}$ Regardless of the type of exercise, it must be intense enough to achieve $80-90 \%$ predicted maximum heart rate, or ventilation corresponding to 17.5-21 times $\mathrm{FEV}_{1} \cdot{ }^{4}$

Weight-bearing exercise is associated with greater ventilatory demand than weight-supported exercise. ${ }^{7}$ Assuming a critical role for high ventilation in increasing postexercise bronchial hyper-responsiveness, ${ }^{1,2,6-11}$ these findings might explain the higher prevalence of EIB after a treadmill test compared to a cycling test. ${ }^{7,12}$ Laboratorybased tests, however, are not widely available and often require bulky and expensive ergometers. Treadmill tests, in particular, raise additional concerns about children's safety and their ability to exercise steadily at high speeds. ${ }^{13}$ Single-step tests are externally valid and a portable alternative to ergometer-based tests, ${ }^{14-16}$ but the metabolic demands associated with stepping might be considerably lower than those associated with running. ${ }^{17}$ Moreover, the vertical work associated with stepping places a greater burden on lower limb muscles. ${ }^{18,19}$ Thus, a stepping test in children may be limited by leg fatigue before they have reached the high levels of ventilation thought necessary to provoke EIB..$^{20,21}$ It is therefore conceivable that by failing to elicit sufficiently high ventilation, stepping tests might be less sensitive than treadmill tests in detecting EIB. This hypothesis, however, has not been tested in children with asthma.

We prospectively compared the prevalence and severity of EIB in response to stepping and treadmill running as modulated by potential inter-modality differences in ventilation in children with asthma with exercise-related symptoms. To investigate whether sudden versus progressive increases in ventilation would also influence a stepping test's ability to detect EIB, ${ }^{22}$ subjects underwent constant work load and incremental stepping tests. We hypothesized that stepping would induce less EIB due to lower ventilatory requirements compared to running. Moreover, we anticipated that a delay in reaching high levels of ventilation would further reduce the diagnostic yield of the incremental compared to the constant stepping test.

\section{Methods}

This was a prospective, cross-sectional study. Subjects' parents or legal guardians signed informed consent, and

Correspondence: Simone Dal Corso PT PhD, Universidade Nove de Julho, Rua Vergueiro no. 235/249, $2^{\circ}$ subsolo, Liberdade, São Paulo, Brazil 01504050. E-mail: si.dal.corso@gmail.com.

DOI: $10.4187 /$ respcare. 06363

\section{QUICK LOOK}

\section{Current knowledge}

Laboratory-based tests are commonly used to evaluate exercise-induced bronchoconstriction (EIB), but are not widely available, and require bulky and expensive ergometers. Single-step tests are an externally validated and portable alternative to ergometer-based tests.

\section{What this paper contributes to our knowledge}

Constant and incremental stepping tests were roughly equivalent in determining EIB, but both protocols exhibited lower metabolic, ventilatory, and cardiovascular demands than treadmill running. Despite higher EIB prevalence after running, some subjects with a negative treadmill test had a positive stepping test. In such cases, a single-step test might be useful to screen for EIB.

children gave their assent to participate in the study, which was approved by the institutional Research Ethics Board (Ethics Committee of the University Nove de Julho, São Paulo, Brazil).

\section{Study Design, Participants, and Measurements}

Fifity-three children and adolescents (34 boys, age $12 \pm 3$ y) with a diagnosis of asthma and a history of exercise-induced respiratory symptoms followed in the asthma out-patient clinic at the Pediatrics Department of São Paulo School of Medicine, Federal University of São Paulo, Brazil, comprised the study group. Patients were excluded for recent disease exacerbation (within the preceding 6 weeks) as stated in the medical record. Smoking, severe asthma (Global Initiative for Asthma ${ }^{23}$ step 5), presence of other chronic lung diseases, and relevant systemic comorbidities (eg, heart disease, diabetes, and musculoskeletal diseases) were also exclusion factors. Subjects' parents (or legal guardians) were instructed to discontinue shortand long-acting $\beta_{2}$ agonists and oral leukotriene receptor antagonist administration within the timeframe defined by current guidelines. ${ }^{1,2,6,20,21}$ Inhaled steroids were discontinued $24 \mathrm{~h}$ before testing. Subjects were instructed to avoid exercise, heavy meals, and coffee, tea, or soft drinks within this timeframe. ${ }^{1,2,6,20,21}$

\section{Spirometry}

Standard spirometric tests (CPX Ultima, Medical Graphics, St. Paul, Minnesota) were performed in the seated position according to the American Thoracic Society/European Respiratory Society criteria. ${ }^{24}$ The Global Lung 
Function Initiative scale was used to classify spirometric values; $z$ scores $>-1.64$ were considered normal, $z$ scores from -1.64 to -2.5 were considered mild obstruction, $z$ scores from -2.5 to -3.5 were considered moderate obstruction, and $z$ scores $<-3.5$ were considered severe obstruction. ${ }^{25}$ Volumes and flows were measured before the challenges and at 5, 10, 15, 20, and $30 \mathrm{~min}$ after the challenges. A $\mathrm{FEV}_{1}$ decrease (nadir) $\geq 10 \%$ from preexercise values defined a positive EIB test. ${ }^{1,2,6}$ EIB was classified as mild (10-15\% $\mathrm{FEV}_{1}$ decrease), moderate (16$25 \%$ decrease), or severe EIB ( $>25 \%$ decrease). ${ }^{2}$ The area under the $\mathrm{FEV}_{1}$ curve (AUC), which provided an additional index of EIB severity, was calculated as the integral of the $\mathrm{FEV}_{1}$ response (\% from pre-exercise) from $5 \mathrm{~min}$ to $30 \mathrm{~min}$ post-challenge (SigmaPlot, version 12.0, Systat Software, San Jose, California).

\section{Exercise Challenges Testing}

Exercise challenge tests were carried out at least $72 \mathrm{~h}$ apart in a randomized sequence. Tests were performed at room temperature between $19^{\circ} \mathrm{C}$ and $24^{\circ} \mathrm{C}$ and relative humidity of $60 \pm 3 \%$. Standard variables including metabolic demand (oxygen consumption $\left(\dot{\mathrm{V}}_{\mathrm{O}_{2}}, \mathrm{~L} / \mathrm{min}\right)$, carbon dioxide output $\left(\dot{\mathrm{V}}_{\mathrm{CO}_{2}}, \mathrm{~L} / \mathrm{min}\right.$ ), ventilatory (minute ventilation $\left(\dot{\mathrm{V}}_{\mathrm{E}}, \mathrm{L} / \mathrm{min}\right)$, cardiovascular demand (heart rate, beats/min), gas exchange $\left(\mathrm{S}_{\mathrm{pO}_{2}}, \%\right)$ were recorded throughout the tests. Subjects were asked to rate dyspnea and leg fatigue at exercise cessation by using the $0-10$ Borg scale. An index of ventilatory demand relative to maximum capacity was established by the $\dot{V}_{\mathrm{E}}$ at the peak of exercise divided by maximum voluntary ventilation (MVV) multiplied by 100. MVV was calculated as pre-exercise $\mathrm{FEV}_{1} \times 40 . \dot{\mathrm{V}}_{\mathrm{E}} / \mathrm{MVV}$ values $\geq 40 \%$ and $\geq 60 \%$ indicated high and very-high ventilatory demands, respectively. ${ }^{1,2,6}$ The $\dot{V}_{\mathrm{E}} / \mathrm{MVV}$ AUC was calculated to provide a more precise index of ventilatory stress. It was separately calculated as the integral over exercise time under total ventilation, high ventilation, and very-high ventilation (SigmaPlot, version 12.0).

\section{Treadmill Test}

The treadmill test (Millenium Classic, Inbrasport, Porto Alegre, Brazil) was performed as proposed by Carlsen et $\mathrm{al}^{26}$ at a $5.5 \%$ grade with speed adjusted within the initial $3 \mathrm{~min}$ to reach $80-90 \%$ maximum predicted heart rate, which was then maintained for 4-6 min.

\section{Stepping Tests}

The single-step tests were performed using a step $15 \mathrm{~cm}$ high for subjects up to $1.40 \mathrm{~m}$ and a step $20 \mathrm{~cm}$ high for taller subjects $\left(>1.40 \mathrm{~m}\right.$ high). ${ }^{15,27}$ The arms were kept unsupported. The standardized instructions recommended by the American Thoracic Society for the 6-min walking test were provided prior to the constant stepping test. ${ }^{28}$ Briefly, subjects were instructed to step up and down at their own cadence during this timeframe. Subjects were allowed to stop at their will and resume exercise regardless of the elapsed time. The same encouragement phrases were provided every minute in an even tone. ${ }^{28}$ During the incremental stepping test, stepping cadence was made faster in response to an external sound stimuli as established by Sykes $^{29}$ and modified by Dal Corso et al, ${ }^{30}$ starting at 10 steps/min, with the stepping rate increased 2 steps/min every $30 \mathrm{~s}$ up to symptom limitation or inability to maintain the required stepping rate for $15 \mathrm{~s} .{ }^{30}$ Objective and subjective criteria for test interruption were the same as those recommended by the American Heart Association for the pediatric population. ${ }^{31}$

\section{Statistical Analysis}

The sample size was calculated to detect a treadmillstep difference in $\mathrm{FEV}_{1} \%$ fall $>3.3 \%$ with a SD of $5.9 \%{ }^{16}$ assuming a $\alpha$ error of $5 \%$ and a $\beta$ error of $20 \%$. These criteria yielded a sample size of 48 subjects. To account for potential dropouts, we assessed 53 children. According to data distribution, measures of central tendency and dispersion were mean $\pm \mathrm{SD}$ or median (interquartile range). Between-group comparisons over time were performed with repeated measurements of analysis of variance (ANOVA). Chi-square tests examined potential differences in proportions. The level of between-test agreement in eliciting EIB was tested by Cohen's kappa $(\leq 0$ indicating no agreement, $0.01-0.20$ as none to slight, $0.21-$ 0.40 as poor, $0.41-0.60$ as moderate, $0.61-0.80$ as substantial, and $0.81-1.00$ as almost perfect agreement). The Bland-Altman test examined between-test differences in EIB severity as a function of their means. The Pearson correlation coefficient was used to test the presence of linear association between continuous variables. A 2 -sided $P$ value $<.05$ was considered statistically significant.

\section{Results}

Sixty-three patients were screened for study participation. Ten patients were excluded ( 2 due to recent exacerbation, 6 due to lack of disease control as indicated by the accompanying physician, and 2 due to lack of understanding of study procedures). Thus, 53 subjects were enrolled (Table 1).

Baseline spirometry indicated the presence of air flow obstruction in 15 subjects ( 8 mild, 6 moderate, 1 severe) with the remaining tests within the limits of normal. Prechallenge spirometric values did not differ among tests. 
Table 1. Subject Characteristics

\begin{tabular}{lc}
\hline \hline \multicolumn{1}{c}{ Variables } & $\begin{array}{c}\text { Values } \\
(n=53)\end{array}$ \\
\hline Age, $\mathrm{y}$ & $12 \pm 3$ \\
Weight, $\mathrm{kg}$ & $44 \pm 13$ \\
Height, cm & $149 \pm 16$ \\
Male/female, $n$ & $34 / 19$ \\
BMI, kg/m ${ }^{2}$ & $19(16-21)$ \\
GINA treatment step 1/2/3/4, $n$ & $19 / 5 / 16 / 13$ \\
Medication use, $n$ & \\
Not taking any medication & 11 \\
SABA & 8 \\
Low dose of ICS & 5 \\
Low dose of ICS + SABA & 6 \\
Medium dose of ICS & 5 \\
Medium dose of ICS + SABA & 3 \\
Low dose of LABA & 2 \\
Medium dose of ICS + LABA & 2 \\
Medium dose of LABA & 3 \\
Medium dose of LABA + SABA & 1 \\
Low/medium dose of ICS + LABA & 4 \\
Medium/high dose ICS + LABA + SABA & 3 \\
& \\
\hline Values are expressed as mean \pm standard deviation. & \\
BMI = body mass index \\
GINA = Global Inititive for Asthma \\
SABA = short-acting $\beta_{2}$ agonists \\
ICS = inhaled corticosteroids \\
LABA = long-acting $\beta_{2}$ agonists & \\
\hline
\end{tabular}

Table 2. Pre Challenge Spirometric Values

\begin{tabular}{|c|c|c|c|}
\hline Variables & Treadmill & $\begin{array}{c}\text { Constant } \\
\text { Stepping } \\
\text { Test }\end{array}$ & $\begin{array}{c}\text { Incremental } \\
\text { Stepping } \\
\text { Test } \\
\end{array}$ \\
\hline FVC, L & $2.7 \pm 1.0$ & $2.8 \pm 1.0$ & $2.7 \pm 1.0$ \\
\hline FVC, $\%$ & $104 \pm 13$ & $103 \pm 14$ & $104 \pm 14$ \\
\hline $\mathrm{FEV}_{1}, \mathrm{~L}$ & $2.2 \pm 0.7$ & $2.2 \pm 0.7$ & $2.2 \pm 0.7$ \\
\hline $\mathrm{FEV}_{1}, \%$ & $82 \pm 10$ & $93 \pm 13$ & $92 \pm 14$ \\
\hline $\mathrm{FEV}_{1} / \mathrm{FVC}, \%$ & $82 \pm 10$ & $83 \pm 10$ & $82 \pm 10$ \\
\hline Peak expiratory flow, L & $4.8 \pm 1.6$ & $4.8 \pm 1.6$ & $4.8 \pm 2.0$ \\
\hline $\mathrm{FEF}_{25-75}, \mathrm{~L}$ & $2.3 \pm 1.0$ & $2.2 \pm 0.8$ & $2.3 \pm 0.8$ \\
\hline $\mathrm{FEF}_{25-75}, \%$ predicted & $100 \pm 38$ & $98 \pm 36$ & $99 \pm 36$ \\
\hline \multicolumn{4}{|c|}{$\begin{array}{l}\mathrm{FEV}_{1}=\text { forced expiratory volume in one second } \\
\mathrm{FEF}_{25-75}=\text { forced expiratory flow between } 25 \% \text { and } 75 \% \text { of } \mathrm{FVC}\end{array}$} \\
\hline
\end{tabular}

Prechallenge spirometric values did not differ among tests (Table 2).

All subjects successfully completed all proposed tests without complications. To reach the target heart rate (80$90 \%$ predicted), ${ }^{26}$ subjects ran during the treadmill tests $(7 \pm 1 \mathrm{~km} / \mathrm{h})$. As expected by the test's design, the constant stepping test was shorter than the treadmill test, whereas the incremental stepping test was longer than the treadmill test (Table 3). The total number of steps performed in the constant stepping test was lower than that in the incremental stepping test $(180 \pm 24$ steps and $292 \pm 86$ steps, respectively). Stepping was associated with lower metabolic, ventilatory, and cardiovascular demand than treadmill running (Fig. 1 for a representative subject). In fact, a greater fraction of subjects reached high $\left(40-60 \% \dot{\mathrm{V}}_{\mathrm{E}} / \mathrm{MVV}\right)$ and very high $\left(60 \% \dot{\mathrm{V}}_{\mathrm{E}} / \mathrm{MVV}\right)$ ventilatory demand during the latter test. The oxygen saturation peak was lower in the treadmill test than in the stepping tests. In contrast, end-exercise symptoms did not differ (Table 3).

Forty-five subjects (85\%) presented with EIB in at least one challenge. Although EIB prevalence was higher after the treadmill test $(60 \%)$ compared to the constant and incremental stepping tests (53\% and $47 \%$, respectively), between-test agreement in diagnosing EIB was poor (Cohen's kappa values were 0.217-0.361) (Table 4). As shown Supplemental Figure 1, and E-Table 1, there were no significant between-test differences in EIB severity. There were no significant clinical and resting functional differences in subjects with EIB negative in a given test but positive in another versus concordant subjects (ie, EIB negative or positive in both tests) (see the supplementary materials at http://www.rcjournal.com).

Figure 2 shows that the presence of EIB was largely independent of the ventilatory demand reached in each of the challenges. For instance, a similar fraction of subjects presented with EIB regardless of high or very-high ventilatory demands attained (24\% and 37\%, respectively) during the treadmill test (Fig. 2A). Similarly, subjects presented with EIB despite test failure in eliciting very-high ventilatory demands (constant stepping test) or even high ventilatory demands (incremental stepping test) (see Figs. 2B and 2C, respectively). Using heart rate as a surrogate of exercise intensity produced similar results.

\section{Discussion}

Our aim was to compare the prevalence and severity of EIB in response to stepping tests and treadmill running tests as modulated by potential inter-modality differences in ventilation in asthmatic children. We hypothesized that stepping would induce less EIB due to lower ventilatory requirements compared to running.

This study contrasted the ventilatory demand elicited by single-step (constant stepping and incremental stepping) and treadmill running challenges as related to their diagnostic yields for EIB in children with asthma and exerciserelated symptoms. We found poor between-test agreement in diagnosing EIB. Thus, despite higher EIB prevalence after running, some subjects with a negative treadmill test had a positive stepping test. In line with our main hypothesis, stepping was associated with lower ventilatory de- 
Table 3. Responses to Running (Treadmill Test) and Stepping (Constant and Incremental Tests) in Asthmatic Children

\begin{tabular}{|c|c|c|c|}
\hline & Treadmill Test & Constant Step Test & Incremental Step Test \\
\hline \multicolumn{4}{|l|}{ Test details } \\
\hline Temperature, $\mathrm{C}^{\circ}$ & $18.0 \pm 0.6$ & $18.0 \pm 0.6$ & $18.0 \pm 0.6$ \\
\hline Humidity, \% & $59 \pm 2$ & $59 \pm 2$ & $59 \pm 2$ \\
\hline Test duration, min & $9 \pm 0 * \dagger$ & $6 \pm 0 \dagger$ & $13 \pm 2$ \\
\hline \multicolumn{4}{|l|}{ Metabolic } \\
\hline$\dot{\mathrm{V}}_{\mathrm{O}_{2}}, \mathrm{~L} / \mathrm{min}$ & $1.65 \pm 0.60 * \dagger$ & $1.42 \pm 0.48$ & $1.34 \pm 0.55$ \\
\hline$\dot{\mathrm{V}}_{\mathrm{CO}_{2}}, \mathrm{~L} / \mathrm{min}$ & $1.76 \pm 0.67 * \dagger$ & $1.47 \pm 0.54$ & $1.42 \pm 0.63$ \\
\hline \multicolumn{4}{|l|}{ Ventilatory and gas exchange } \\
\hline$\dot{\mathrm{V}}_{\mathrm{E}}, \mathrm{L} / \mathrm{min}$ & $54 \pm 17 * \dagger$ & $45 \pm 14$ & $43 \pm 16$ \\
\hline$\dot{\mathrm{V}}_{\mathrm{E}} / \mathrm{MVV}$ & $0.62 \pm 0.13 * \dagger$ & $0.52 \pm 0.14$ & $0.49 \pm 0.11$ \\
\hline AUC $\dot{V}_{E} / M V V$, a.u. & $401 \pm 84^{* \dagger}$ & $226 \pm 53 \dagger$ & $345 \pm 87$ \\
\hline$\dot{\mathrm{V}}_{\mathrm{E}} / \mathrm{MVV} \geq 40 \%, n(\%)$ & $52(98 \%) \dagger$ & $44(83 \%) \dagger$ & $32(60 \%)$ \\
\hline AUC $\dot{V}_{E} / M V V \geq 40 \%$, a.u. & $323 \pm 112 * \dagger$ & $166 \pm 14$ & $137 \pm 14$ \\
\hline$\dot{\mathrm{V}}_{\mathrm{E}} / \mathrm{MVV} \geq 60 \%, n(\%)$ & $33(62 \%) * \dagger$ & $13(24 \%) \dagger$ & $3(6 \%)$ \\
\hline AUC $\dot{V}_{E} / M V V \geq 60 \%$, a.u. & $257 \pm 141 * \dagger$ & $142 \pm 93$ & $64 \pm 74$ \\
\hline $\mathrm{S}_{\mathrm{pO}_{2}}, \%$ & $93 \pm 3 \dagger$ & $94 \pm 3$ & $95 \pm 3$ \\
\hline \multicolumn{4}{|l|}{ Cardiovascular } \\
\hline Heart rate, beats/min & $172 \pm 7 * \dagger$ & $160 \pm 20$ & $161 \pm 19$ \\
\hline Heart rate, $\%$ predicted & $86 \pm 3^{* \dagger}$ & $80 \pm 10$ & $81 \pm 10$ \\
\hline AUC heart rate, a.u. & $714 \pm 38 * \dagger$ & $441 \pm 44$ & $834 \pm 210$ \\
\hline Heart rate $\geq 80 \%, n(\%)$ & 53 & 25 & 34 \\
\hline AUC heart rate $\geq 80 \%$, a.u. & $714 \pm 38 * \dagger$ & $288 \pm 144$ & $239 \pm 243$ \\
\hline Heart rate $\geq 90 \%, n(\%)$ & 0 & 10 & 10 \\
\hline AUC heart rate $\geq 90 \%$, a.u. & 0 & $217 \pm 173$ & $219 \pm 210$ \\
\hline \multicolumn{4}{|l|}{ Symptoms } \\
\hline Borg dyspnea score & $4(2-6)$ & $4(1-6)$ & $3(1-5)$ \\
\hline Borg leg effort score & $5(3-7)$ & $4(2-7)$ & $3(2-5)$ \\
\hline \multicolumn{4}{|l|}{ Post-exercise } \\
\hline Nadir $\mathrm{FEV}_{1}$ fall, $\%$ & $-16 \pm 15$ & $-12 \pm 9$ & $-14 \pm 14$ \\
\hline EIB (mild, moderate, severe) & $20 / 10 / 2$ & $23 / 5 / 0$ & $16 / 7 / 2$ \\
\hline AUC FEV ${ }_{1} \%$ fall, a.u. & $-250 \pm 334$ & $-178 \pm 196$ & $-210 \pm 321$ \\
\hline $\begin{array}{l}\text { Values were measure at end of exercise unle } \\
* P<.05 \text { compared to constant step. } \\
\dagger P<.05 \text { compared to incremental step. } \\
\dot{\mathrm{V}}_{\mathrm{O}_{2}}=\text { oxygen consumption } \\
\dot{\mathrm{V}}_{\mathrm{CO}_{2}}=\text { carbon dioxide production } \\
\dot{\mathrm{V}}_{\mathrm{E}}=\text { minute ventilation } \\
\mathrm{MVV}=\text { maximum voluntary ventilation } \\
\mathrm{AUC}=\text { area under the curve } \\
\mathrm{EIB}=\text { exercise-induced bronchoconstriction }\end{array}$ & Values are mean $\pm \mathrm{SD}$ & range) unless otherwise note & \\
\hline
\end{tabular}

mands than running. Contrary to our hypothesis, however, EIB prevalence and severity were largely independent of between-test differences in ventilatory demand. Moreover, the rate of increase in ventilation (ie, faster in constant stepping compared to incremental stepping) did not influence test outcome. Our findings therefore indicate that the ability to elicit high ( $\geq 40 \% \mathrm{MVV}$ ) or very-high ( $\geq 60 \% \mathrm{MVV}$ ) ventilation is not a key requirement for an effective EIB challenge in children with asthma with a high pre-test likelihood of abnormality. Although a stepping test (either constant or incremental) can be used to screen for EIB, a negative challenge should be confirmed with a high-intensity treadmill test. Single-step tests have long been advocated as a portable, inexpensive, and reproducible field exercise testing modality for patients with chronic lung diseases. ${ }^{30,32,33}$

In this study, we showed that stepping is associated with less ventilatory stress than treadmill running in children with asthma. A substantial fraction of subjects did not reach the ideal ventilatory stress recommended by current guidelines in response to stepping (Fig. 2).1,2,6 These findings could explain the lower prevalence of EIB that we observed (Table 4). Contrary to our expectations, we were unable to find supporting evidence that lower ventilatory stress during stepping hampered test sensitivity in detecting EIB. Thus, our results indicate that reaching high to very-high ventilatory demands is not required to elicit EIB 

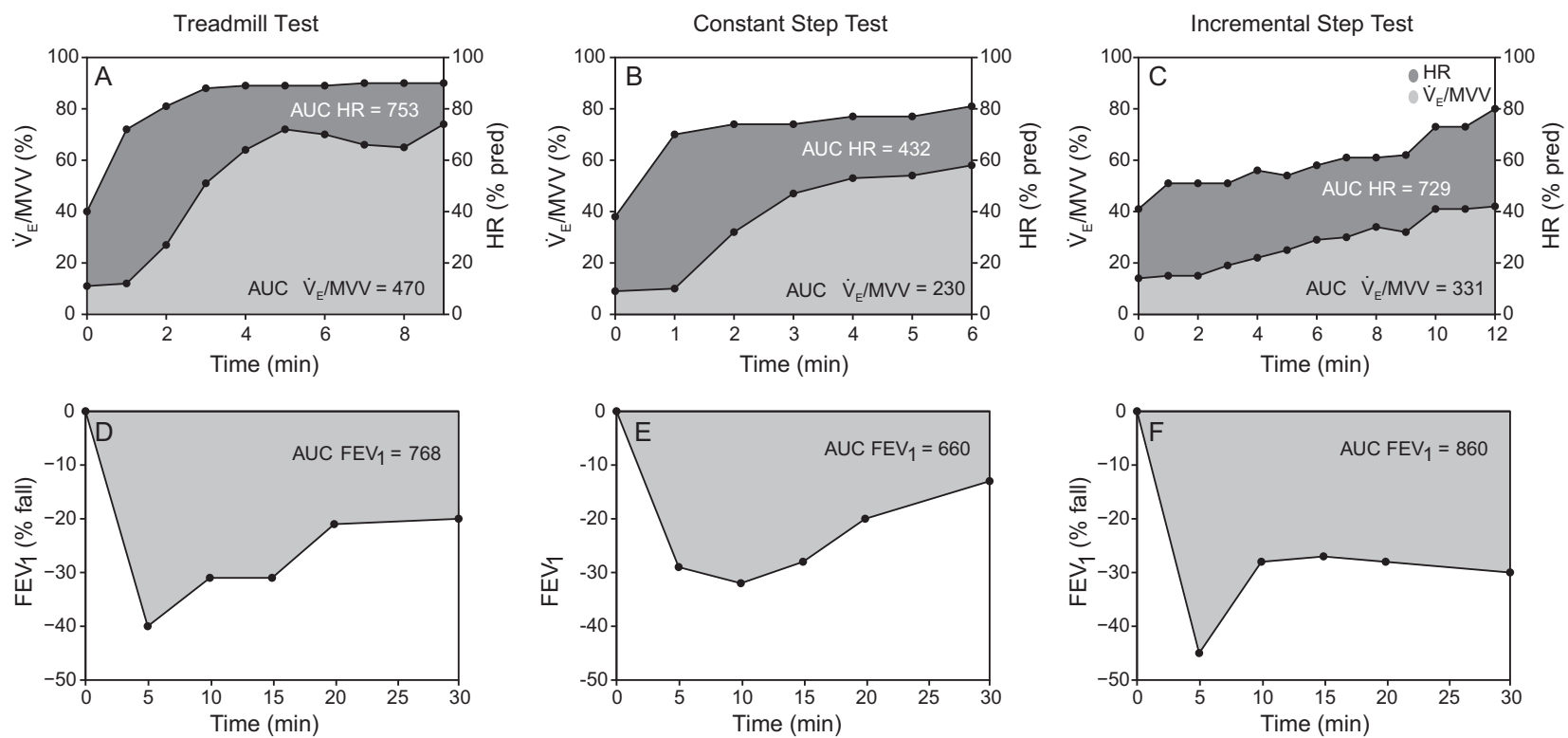

Fig. 1. Representative ventilatory and cardiovascular responses during different exercise challenges in a subject with positive exerciseinduced bronchoconstriction $(\mathrm{A}-\mathrm{C})$. Panels $\mathrm{D}-\mathrm{F}$ depict the resulting post-exercise decreases in $\mathrm{FEV}_{1} \cdot \dot{\mathrm{V}}_{\mathrm{E}}=$ minute ventilation, $\mathrm{MVV}=$ maximum voluntary ventilation, $\mathrm{AUC}=$ area under the curve, $\mathrm{HR}=$ heart rate.

Table 4. Between-Test Agreement for the Diagnosis of EIB in Asthmatic Children

\begin{tabular}{|c|c|c|c|}
\hline & $\operatorname{EIB}(+)$ & EIB $(-)$ & Total \\
\hline \multicolumn{4}{|l|}{ Treadmill test } \\
\hline \multicolumn{4}{|c|}{ Constant step test, $n(\%)$} \\
\hline $\mathrm{EIB}(+)$ & $21(40)^{*}$ & $7(13)$ & $28(53)$ \\
\hline $\operatorname{EIB}(-)$ & $11(21)$ & $14(26)^{*}$ & $25(47)$ \\
\hline \multicolumn{4}{|c|}{ Incremental step test, $n(\%)$} \\
\hline EIB $(+)$ & $18(34)^{*}$ & $7(13)$ & $25(47)$ \\
\hline EIB $(-)$ & $14(26)$ & $14(26)^{*}$ & $28(53)$ \\
\hline Total & $32(60)$ & $21(40)$ & 53 \\
\hline \multicolumn{4}{|c|}{ Constant step test } \\
\hline \multicolumn{4}{|c|}{ Incremental step test, $n(\%)$} \\
\hline $\mathrm{EIB}(+)$ & $18(34)^{*}$ & $7(13)$ & $25(47)$ \\
\hline EIB $(-)$ & $10(19)$ & $18(34)^{*}$ & $28(53)$ \\
\hline Total, $n(\%)$ & $28(53)$ & $25(47)$ & 53 \\
\hline \multicolumn{4}{|c|}{$\begin{array}{l}\text { * Kappa coefficient ( } \pm \text { standard error): treadmill vs constant step test: } 0.313 \pm 0.130 \text {; } \\
\text { treadmill vs incremental step test: } 0.217 \pm 0.129 \text {; constant vs incremental step test: } \\
0.361 \pm 0.127 \text {. } \\
\text { EIB }=\text { exercise-induced bronchoconstriction }\end{array}$} \\
\hline
\end{tabular}

in highly susceptible patients, that is, in children with asthma with a history of exercise-related symptoms. Due to the large day-to-day variability in EIB, repeating the challenge might be more relevant to improve diagnostic yield than increasing testing intensity. ${ }^{34}$

Nevertheless, failure to reach such high targets might be critical for children with lower pre-test probability of EIB, such as non-asthmatic children without exercise-related symptoms. Due to the lack of differences in environmental conditions and ventilatory demands in patients who developed EIB only after treadmill, alternative explanations should be sought to explain the higher sensitivity of running to elicit EIB. Interestingly, seminal studies presented evidence that the asthmogenic properties of running were not a mere consequence of a higher ventilation load. ${ }^{11,12,35}$ In this context, oral breathing is greater in running compared to walking, ${ }^{36}$ so it is possible that the former is associated with less air humidification. ${ }^{37}$ Release of mediators by the distal (small) airways is mechanistically linked to EIB. ${ }^{20}$

Differences in breathing pattern and ventilation distribution might expose a larger fraction of the small airways to drier inspired air during running. Moreover, higher cardiac output and greater bronchial blood flow during running might increase local availability of bronchoconstrictive mediators. ${ }^{38}$ Regardless of the precise mechanisms, our data indicate that the superiority of running compared to stepping in eliciting EIB is not simply consequence of high ventilatory demands inherent to upright, weight-bearing exercise. From a practical perspective, our results demonstrate that treadmill and stepping tests are not interchangeable as exercise challenges for EIB. Specifically, a negative stepping test should be confirmed with a highintensity treadmill test, particularly in a patient with a high pre-test likelihood of abnormality. Surprisingly, however, a fraction of our subjects presented with EIB only in response to stepping (Table 4).

We cannot rule out that a negative treadmill test with a positive stepping test merely reflects day-to-day variabil- 

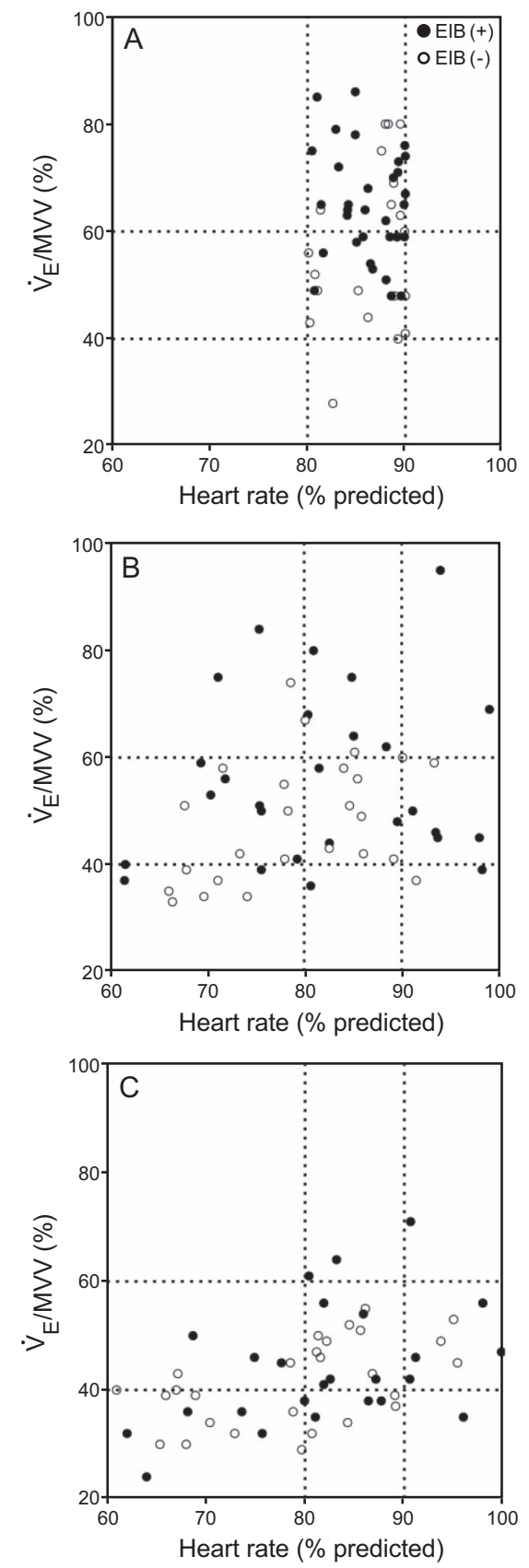

Fig. 2. Ventilatory and cardiovascular demand in response to different exercise challenges in subjects who presented with $(+)$ or without (-) EIB. (A) Treadmill test. (B) Constant step test. (C) Incremental step test. $\dot{\mathrm{V}}_{\mathrm{E}}=$ minute ventilation, $\mathrm{MVV}=$ maximum voluntary ventilation, EIB = exercise-induced bronchoconstriction.

ity in EIB susceptibility. ${ }^{2}$ In other words, it remains unclear whether these subjects would eventually develop EIB had we repeated the treadmill test. ${ }^{38}$ Additional studies investigating this specific issue are warranted. It should be recognized specifically that the incremental stepping test adds complexity to a field challenge. Considering that the diagnostic yields of constant and incremental stepping tests were roughly equivalent (Table 4), the former seems to be a clinically more attractive option. Whether shorter tests (eg, $3 \mathrm{~min})^{15}$ would also prove useful remains to be investigated in this specific sub-population of children with exercise-related symptoms.

This study has some limitations. We did not test our subjects on the treadmill at the same (lower) ventilatory demands reached in the stepping tests. Thus, we cannot elaborate on whether low to moderate ventilatory demands would also be effective in eliciting EIB in this specific test modality. Exercise load in the treadmill test was guided by a physiological outcome (heart rate) that reflects exercise intensity. In contrast, the stepping tests were either selfpaced or externally paced. Thus, it could be argued that our study design biased the treadmill test to elicit EIB more frequently.

As discussed, however, our data do not indicate that reaching a high cardiorespiratory stress was critical for EIB in our sample. Under the logical assumption that there is a minimal ventilatory load associated with EIB, prospective studies are necessary to investigate whether such requirements are modulated by exercise modality.

\section{Conclusion}

The lower prevalence of EIB in stepping tests compared to treadmill running tests was not related to less ventilatory demand in the former testing modality. Regarding the chosen challenge, testing ability to elicit high or very-high ventilation was not a key feature of an effective EIB test in children with asthma with a history of exercise-related symptoms. Although a single-step test might be useful to screen for EIB, a negative challenge should be confirmed with a running-based test in this patient sub-population.

\section{ACKNOWLEDGMENTS}

We thank Anderson Jose, Laryssa Souza Leitão, Felipe Elias Moreira, and Mariana Mazucca Reimberg (Postgraduate Program in Rehabilitation Sciences, Universidade Nove de Julho, São Paulo, Brazil) for helping with subject recruitment and evaluations.

\section{REFERENCES}

1. Crapo RO, Casaburi R, Coates AL, Enright PL, Hankinson JL, Irvin CG, et al. Guidelines for methacholine and exercise challenge testing 1999. Am J Respir Crit Care Med 2000;161(1):309-329.

2. Weiler JM, Brannan JD, Randolph CC, Hallstrand TS, Parsons J, Silvers W, et al. Exercise-induced bronchoconstrictionupdate - 2016. J Allergy Clin Immunol 2016;138(5):1292-1295.

3. de Aguiar KB, Anzolin M, Zhang L. Global prevalence of exerciseinduced bronchoconstriction in childhood: a meta-analysis. Pediatr Pulmonol. 2018;53(4):412-425.

4. Parsons JP, Hallstrand TS, Mastronarde JG, Kaminsky DA, Rundell KW, Hull JH, et al. An official American Thoracic Society clinical practice guideline: exercise-induced bronchoconstriction. Am J Respir Crit Care Med 2013;187(9):1016-1027. 


\section{Ventilatory Demand During Exercise}

5. Parsons JP, Baran CP, Phillips G, Jarjoura D, Kaeding C, Bringardner B, et al. Airway inflammation in exercise-induced bronchospasm occurring in athletes without asthma. J Asthma 2008;45(5):363-367.

6. De Baets F, Bodart E, Dramaix-Wilmet M, Van Daele S, de Bilderling G, Masset S, Vermeire P, Michel O. Exercise-induced respiratory symptoms are poor predictors of bronchoconstriction. Pediatr Pulmonol 2005;39(4):301-305.

7. ERS Task Force, Palange P, Ward SA, Carlsen KH, Casaburi R, Gallagher CG, et al. Recommendations on the use of exercise testing in clinical practice. Eur Respir J 2007;29(1):185-209.

8. Smoliga JM, Weiss P, Rundell KW. Exercise-induced bronchoconstriction in adults: evidence based diagnosis and management. BMJ 2016;352:h6951.

9. Astrand P, Rodahl K. Textbook of work physiology, 3rd edition. New York: McGraw-Hill; 1986.

10. Kivity S, Souhrada JF, Melzer E. A dose-response-like relationship between minute ventilation and exercise-induced bronchoconstriction in young asthmatic patients. Eur J Respir Dis 1980;61(6):342346.

11. Anderson SD, Connolly NM, Godfrey S. Comparison of bronchoconstriction induced by cycling and running. Thorax 1971;26(4):396401.

12. Godfrey S, Silverman M, Anderson SD. The use of the treadmill for assessing exercise-induced asthma and the effect of varying the severity and duration of exercise. Pediatrics 1975;56(5 pt22 suppl): 893-898.

13. Cordingley D, Girardin R, Reimer K, Ritchie L, Leiter J, Russell K, Ellis MJ. Graded aerobic treadmill testing in pediatric sports-related concussion: safety, clinical use, and patient outcomes. J Neurosurg Pediatr 2016;25(6):693-702.

14. Francis K, Feinstein R. A simple height-specific and rate-specific step test for children. South Med J 1991;84(2):169-174.

15. Feinstein RA, Hains CS, Hemstreet MP, Turner-Henson A, Redden DT, Martin B, et al. A simple "step-test" protocol for identifying suspected unrecognized exercise-induced asthma (EIA) in children. Allergy Asthma Proc 1999;20(3):181-188.

16. Tancredi G, Quattrucci S, Scalercio F, De Castro G, Zicari AM, Bonci E, et al. 3-min step test and treadmill exercise for evaluating exercise-induced asthma. Eur Respir J 2004;23(4):569-574.

17. Buckley JP, Sim J, Eston RG, Hession R, Fox R. Reliability and validity of measures taken during the Chester step test to predict aerobic power and to prescribe aerobic exercise. Br J Sports Med 2004;38(2):197-205.

18. Scharf-Olson M, Williford HN, Blessing DL, Moses R, Wang T. Vertical impact forces during bench-step aerobics: exercise rate and experience. Percept Mot Skills 1997;84(1):267-374.

19. Müller PT, Christofoletti G, Zagatto AM, Paulin FV, Neder JA. Reliability of peak $\mathrm{O}_{2}$ uptake and $\mathrm{O}_{2}$ uptake kinetics in step exercise tests in healthy subjects. Respir Physiol Neurobiol 2015;207:7-13.

20. Weiler JM, Anderson SD, Randolph C, Bonini S, Craig TJ, Pearlman DS, et al. Pathogenesis, prevalence, diagnosis, and management of exercise-induced bronchoconstriction: a practice parameter. Ann Allergy Asthma Immunol 2010;105(6 suppl):S1-S47.

21. Del Giacco SR, Firinu D, Bjermer L, Carlsen K-H. Exercise and asthma: an overview. Eur Clin Respir J 2015;2:27984.
22. De Fuccio MB, Nery LE, Malaguti C, Taguchi S, Dal Corso S, Neder JA. Clinical role of rapid-incremental tests in the evaluation of exercise-induced bronchoconstriction. Chest 2005;128(4):2435-2442.

23. Global Initiative for Asthma. Global Strategy for Asthma Management and Prevention, 2018. www.ginasthma.org, Accessed April 16, 2018.

24. Miller MR, Hankinson J, Brusasco V, Burgos F, Casaburi R, Coates A, et al. Standardisation of spirometry. Eur Respir J 2005;26(2):319338.

25. Quanjer PF, Stanojevic S, Cole TJ, Baur X, Hall GL, Culver BH. Multi-ethnic reference values for spirometry for the 3-95-yr age range: the global lung function 2012 equations Eur Respir J 2012; 40:1324-1343.

26. Carlsen KH, Engh G, Mørk M. Exercise-induced bronchoconstriction depends on exercise load. Respir Med 2000;94(8):750-755.

27. Feinstein RA, LaRussa J, Wang-Dohlman A, Bartolucci AA. Screening adolescent athletes for exercise-induced asthma. Clin J Sport Med 1996;6(2):119-123.

28. ATS Committee on Proficiency Standards for Clinical Pulmonary Function Laboratories. ATS statement: guidelines for the six-minute walk test. Am J Respir Crit Care Med 2002;166(1):111-117.

29. Sykes K. Capacity assessment in the workplace: a new step test. Occup Health J Occup Health Nurses 1995;47(1):20-22.

30. Dal Corso S, de Camargo AA, Izbicki M, Malaguti C, Nery LE. A symptom-limited incremental step test determines maximum physiological responses in patients with chronic obstructive pulmonary disease. Respir Med 2013;107(12):1993-1999.

31. Paridon SM, Alpert BS, Boas SR, Cabrera ME, Caldarera LL, Daniels SR, et al. Clinical stress testing in the pediatric age group: a statement from the American Heart Association Council on Cardiovascular Disease in the Young, Committee on Atherosclerosis, Hypertension, and Obesity in Youth. Circulation 2006;113(15):19051920

32. Dal Corso S, Duarte SR, Neder JA, Malaguti C, de Fuccio MB, de Castro Pereira CA, Nery LE. A step test to assess exercise-related oxygen desaturation in interstitial lung disease. Eur Respir J 2007; 29(2):330-336

33. Stephan S, Castro Pereira CA de, Coletta EM, Ferreira RG, Otta JS, Nery LE. Oxygen desaturation during a 4-minute step test: predicting survival in idiopathic pulmonary fibrosis. Sarcoidosis Vasc Diffuse Lung Dis 2007;24(1):70-76.

34. Anderson SD, Pearlman DS, Rundell KW, Perry CP, Boushey H, Sorkness CA, et al. Reproducibility of the airway response to an exercise protocol standardized for intensity, duration, and inspired air conditions, in subjects with symptoms suggestive of asthma. Respir Res 2010;11:120.

35. Anderson SD, Silverman M, Tai E, Godfrey S. Specificity of exercise in exercise-induced asthma. BMJ 1971;4(5790):814-815.

36. Morton AR, King K, Papalia S, Goodman C, Turley KR, Wilmore $\mathrm{JH}$. Comparison of maximal oxygen consumption with oral and nasal breathing. Aust J Sci Med Sport 1995;27(3):51-55.

37. Mangla PK, Menon MP. Effect of nasal and oral breathing on exercise-induced asthma. Clin Allergy 1981;11(5):433-439.

38. Duner H, Pernow B. Histamine and leukocytes in blood during muscular work in man. Scand J Clin Lab Invest 1958;10(4):394-396. 